\title{
Reviewing the Clinical Spectrum Related to Kmt2b Gene Mutations: Unusual Clinical Presentation and a Possible New Pathogenic Mutation
}

Cristina del Toro ( $\sim$ cristinadeltoro@msn.com )

Torrecárdenas Hospital Complex: Complejo Hospitalario Torrecardenas https://orcid.org/0000-00030051-9649

\section{Jesús Olivares Romero}

Torrecárdenas Hospital Complex: Complejo Hospitalario Torrecardenas

\section{Research Article}

Keywords: KMT2B, dystonia, unusual clinical presentation, variant of uncertain significance, missense, adult onset, focal dystonia, generalizaed dystonia, case report

Posted Date: December 28th, 2021

DOl: https://doi.org/10.21203/rs.3.rs-878780/v1

License: (c) (i) This work is licensed under a Creative Commons Attribution 4.0 International License. Read Full License 


\section{Abstract}

\section{Introduction}

KMT2B related dystonia is a childhood onset generalized dystonia. Since its first description in 2016, different phenotypic spectrum have been reported. The aim of the case report is to provide data that may help to understand the spectrum of KMT2B-related disorders. We present two members of a family with a possible non-previously described pathogenic mutation and an unusual KMT2B related dystonia presentation: an adult onset and focal dystonia.

\section{Case Presentation}

The index patient is a 32 year-old woman with a generalized dystonia. Her maternal uncle presented a focal dystonia. Next-generation sequencing revealed a heterozygous missense mutation in KMT2B gene (19q13.12), described as a variant of uncertain significance (VUS).

Although characteristic phenotype of KMT2B dystonia is a childhood onset generalized dystonia, different phenotypes have been related according to the kinds of mutations in this gene, also varying the age of symptom onset and the penetrance of the mutation. Asymptomatic or sub-clinical carriers and adult onset has been described. Due to the low prevalence of this variant in the general population and the low penetrance and high intrafamilial variability of this entity, we suggest that this mutation might be a pathogenic variant.

\section{Conclusions}

KMT2B related dystonia is an emerging and prevalent monogenic dystonia whose incidence, genetic variability and clinical spectrum remain unknown. Despite the study of this gene is indicated in childhood onset dystonia, description of cases such as ours shows that its sequencing in patients with an adultonset dystonia with family history can be useful for the diagnosis.

\section{Introduction}

KMT2B related dystonia, also known as DYT 28 , is a childhood onset movement disorder characterized by lower limb dystonia that progresses to a generalized dystonia with cervical, cranial, and laryngeal involvement that causes dysphagia and/or dysphonia. Intellectual impairment associated with facial dysmorphic signs (elongated face with nasal tip) has been described in more than $50 \%$ of reported cases. Other signs and symptoms are less frequently found: short stature, other movement disorders, eye movement abnormalities and dermatologic features.(1-3) Psychiatric features are not uncommon. (4) Image findings are present in $25 \%$ of patients with symmetrical hypointensity of the globus pallidi on diffusion and T2 weighted Magnetic Resonance Image (MRI). $(1,4)$ Treatment options include anticholinergic drugs, botulinum toxin and deep brain stimulation of the globus pallidus internus.(4) 
The aim of the case report is to provide data that may help to understand the spectrum of KMT2B-related disorders. We present two members of a family with a new KMT2B mutation and unusual clinical presentation: adult onset in the index patient and focal dystonia in her family.

\section{Case Presentation}

The index patient is a 32 year-old woman with no previous medical history with the exception of anxiety disorder. She presented an insidious walking difficulty secondary to a dystonic posture aggravated by ambulation with left hip adduction, clubfoot, and ipsilateral scapular elevation that progressed during the following five years, affecting ocular movements and causing dysphagia and hypophonic speech. On directed anamnesis she related a maternal uncle (82 year-old) with a childhood-onset static torticollis. Cognitive function was not affected. Additional tests including an extensive blood test, electromyography and complete column and cranial MRI were normal. Since clinic picture suggested a hereditary generalized dystonia, a genetic panel for DYT was performed after obtaining informed consent. Nextgeneration sequencing revealed a heterozygous missense mutation in KMT2B gene (19q13.12) with a change in the nucleotide c.4219 G > A and therefore in the p.Gly1407Arg aminoacid, described as a variant of uncertain significance (VUS). In the light of these findings the study was also performed to the patient's uncle and asymptomatic mother, confirming the same mutation found in the index patient. The rest of the patient's family -including the mother-was healthy. Treatment with botulinum toxin was successfully initiated in the index patient.

KMT2B gene mutation as a cause of dystonia was first described in 2016.(2) Since then, 66 mutations in at least 133 patients have been reported.(3-6) Most of them are de novo, embracing delections, nonsense, splice-sice and missense mutations. $(4,5)$ Our mutation is registered in the database as a variant present in the European population with a prevalence of 0,0004\%.(7) Nevertheless, due to the low prevalence of this variant in the general population and the low penetrance and high intrafamilial variability of this entity, $(2,4,6)$ we suggest that this mutation might be a pathogenic variant.

Patients's phenotypes were markedly different in spite of presenting the same mutation, adding evidence to the penetrance variability of this mutation. Although characteristic phenotype ( $83 \%$ of patients) of the disease is a childhood onset dystonia (mean age at onset $6.4 \pm 5.9$ y.0) with a generalized progression, adult onset has been described with only few cases in the late adulthood. $(1,5,6,8)$ Mutation found in these patients was a missense type, $(3,8)$ same mutation found in our patient and known to be related to a later onset dystonia.(5) Delection mutations are related with a lower age onset of the disease.(8) Also focalized dystonia has been described in two patients of the same family, (2) as our patient's family presented. Asymptomatic mothers can be carriers of the mutation (2) and carrier parents may present sub-clinical disease features and therefore report no symptoms.(8) It has been showed that approximately $9 \%$ of inherited mutations are received from an asymptomatic progenitor, (3) suggesting an incomplete penetrance which is further reduced in missense mutations. (8) Despite it is not infrequent de novo mutations, (4) since some patients with healthy progenitors may have been diagnosed with de 
novo mutations without genetic testing of family members,(4) this rate of inherited KMT2B mutation from asymptomatic carriers could have been underestimated.

Development of next generation sequencing has allowed a better insight to the understanding of genetically determined dystonias, that often present additional neurological and systemic features, being the psychiatric clinic in close relationship with neurological symptomatology. Hyperactivity disorder and anxiety has shown to be frequent in DYT 28,(4) as the index patient presented and hampered the diagnosis. Subtle features of dystonia could have been present in our patient years before referral to specialist, as well as non-motor features such as the anxiety disorder.

The variety in the way this entity develops over the years together with its phenotypic variability add difficulty to a disease that is already a diagnostic challenge, even more so for clinicians who are not movement disorder specialists.

\section{Conclusions}

KMT2B related dystonia is an emerging and prevalent monogenic dystonia whose incidence, genetic variability and semiology remain unknown. Despite the study of this gene is indicated in childhood onset dystonia, description of cases such as ours shows that its sequencing in patients with an adult-onset dystonia with family history can be useful for the diagnosis and further description of KMT2B-related disorders.

\section{Declarations}

\section{i. Funding:}

This work received no funding.

\section{ii. Conflicts of interest/Competing interests:}

This research did not receive any specific grant from funding agencies in the public, commercial, or notfor-profit sectors.

\section{iii. Ethics approval:}

Therapeutic clinical decisions were supported by the best available evidence from health care research.

\section{iv. Consent to participate:}

Consent to participate was obtained from the patient. 


\section{v. Consent for Publication:}

Written informed consent was obtained from the patient for publication of this case report.

\section{vi. Availability of data and material:}

Data sharing not applicable to this article as no datasets were generated or analyzed during the current study.

\section{vii. Code availability (software application or custom code):}

Not applicable.

\section{viii. Authors' contributions:}

CT.P. wrote the Case Report. J.O.R supervised and reviewed the manuscript. All authors have read and agreed to the published version of the manuscript.

\section{References}

1. Meyer E, Carss KJ, Rankin J, Nichols JM, Grozeva D, Joseph AP, et al. Mutations in the histone methyltransferase gene KMT2B cause complex early-onset dystonia. Nat Genet. 2017;49(2):223-37.

2. Zech M, Boesch S, Maier EM, Borggraefe I, Vill K, Laccone F, et al. Haploinsufficiency of KMT2B, Encoding the Lysine-Specific Histone Methyltransferase 2B, Results in Early-Onset Generalized Dystonia. Am J Hum Genet. 2016;99(6):1377-87.

3. Zech M, Lam DD, Winkelmann J. Update on KMT2B-Related Dystonia. Curr Neurol Neurosci Rep. 2019;19(11):92.

4. Cif L, Demailly D, Lin JP, Barwick KE, Sa M, Abela L, et al. KMT2B-related disorders: expansion of the phenotypic spectrum and long-term efficacy of deep brain stimulation. Brain. 2020;143(11):324261.

5. Gorman KM, Meyer E, Kurian MA. Review of the phenotype of early-onset generalised progressive dystonia due to mutations in KMT2B. Eur J Paediatr Neurol. 2018;22(2):245-56.

6. Pandey S, Bhattad S, Panda AK, Mahadevan L. Late-onset KMT2B-related dystonia in an Indian patient with normal cognition, dystonic opisthotonus and lack of oromandibular and laryngeal involvement. Parkinsonism Relat Disord. 2020;74:33-5.

7. 7. Broad institute gnomAD[Internet][Consulted 17 Ago 2020] Accessible on: https://gnomadbroadinstituteorg. 
8. Zech M, Jech R, Havránková P, Fečíková A, Berutti R, Urgošík D, et al. KMT2B rare missense variants in generalized dystonia. Mov Disord. 2017;32(7):1087-91.

\section{Supplementary Files}

This is a list of supplementary files associated with this preprint. Click to download.

- CAREchecklist.kmt2b.pdf 